INTERNACIONAL

\title{
Migrantes en la mira: ¿discriminación estructural en Argentina?
}

\author{
Migrants in the crosshairs: structural discrimination in Argentina?
}

\section{Martina Olivera}

Universidad de Buenos Aires, Argentina

\begin{abstract}
RESUMEN Las personas migrantes constantemente han sido víctimas de discriminación, sobre todo en la actualidad. De hecho, ha sido noticia la legislación regresiva en materia de derechos humanos de las personas migrantes, lo que ha llegado hasta su criminalización. Sin embargo, este no es un fenómeno nuevo en Argentina, sino que se ha ido gestando desde hace décadas para llegar a positivizarse en un Decreto de Necesidad y Urgencia - de dudosa constitucionalidad y convencionalidad-, e incluso a plasmarse en una serie de políticas públicas que ponen a las personas migrantes en la mira, como el «chivo expiatorio» que debe ser expulsado. Desde una perspectiva de derechos humanos, se podría afirmar que actualmente existen políticas de persecución en contra de las personas migrantes con actitudes activas del Estado, incluyendo el dictado de normativa discriminatoria. En este contexto, el artículo se centrará en definir qué se entiende por discriminación estructural desde el Sistema Interamericano de Protección de Derechos Humanos, y analizará las políticas públicas argentinas relacionadas con el fenómeno de la migración bajo el prisma de ese tipo de discriminación. El objetivo es identificar si se puede hablar de discriminación estructural en Argentina.
\end{abstract}

PALABRAS CLAVE Derechos Humanos, migrantes, igualdad y no discriminación, discriminación estructural.

ABSTRACT Migrants have been constantly victims of discrimination, especially in these times. An example of these is the regressive legislation on migrants' rights adopted by Argentina, criminalizing them for the mere fact of being a migrant. However, this is not a new phenomenon in Argentina, it has been brewing for decades until it became written in a Necessity and Urgency Decree of dubious constitutionality and conventionality, as well as a series of public policies that put migrants in the crosshairs, as the «scapegoat» that must be expelled. From a human rights perspective, it could be affirmed that there are currently policies of persecution against migrants with active attitudes of the State, including the enactment of discriminatory regulations. In this 
sense, the article will focus on defining what is the meaning, from the Inter-American System for the Protection of Human Rights perspective, of structural discrimination and will analyzing Argentinean public policies related to migration, under the prism of that type of discrimination, in order to identify if we can affirm that there is structural discrimination against migrants in Argentina.

KEYWORDS Human Rights, migrants, equality and non-discrimination, structural Discrimination.

\section{Introducción}

En el discurso inaugural de la trigésima novena sesión ordinaria del Consejo de Derechos Humanos de la Organización de las Naciones Unidas, la alta comisionada para los derechos humanos Michelle Bachelet mencionó la cuestión migratoria, haciendo especial énfasis en la importancia del Pacto Mundial sobre Migración. ${ }^{1}$

En relación con los Estados de origen de las personas migrantes, la alta comisionada llamó la atención sobre la situación humanitaria que se vive en Nicaragua y en Venezuela. Asimismo, se refirió a los países de recepción como Estados Unidos - que se negó a firmar el Pacto Mundial—, Hungría — que adoptó medidas tendientes a permitir que sus autoridades arresten, imputen y expulsen a cualquier abogado o abogada sospechoso de asistir a una persona que solicite asilo-, e Italia, donde se ha reportado un rápido aumento de actos de violencia y racismo contra las personas migrantes.

En este contexto mundial y regional de crisis de gobernabilidad migratoria, Argentina no debe pasar desapercibida (Vera-Espinoza, Brumat y Geddes, 2017; Domenech, 2007: 11). En este sentido, en los últimos años se han adoptado una serie de políticas públicas que son alarmantes desde una perspectiva de derechos humanos, específicamente en términos de igualdad y no discriminación.

Estos dos conceptos son claves al momento de analizar la situación de una minoría, como las personas migrantes y el punto de partida que se debe utilizar para el análisis de cualquier política pública que se adopte al respecto. Por esto, en la primera sección de este artículo se analizará cómo han sido tratados ambos conceptos por la Corte Interamericana de Derechos Humanos, especialmente cuál ha sido la definición de discriminación adoptada por dicho tribunal, teniendo en cuenta que la Convención Americana sobre Derechos Humanos no posee una definición propia.

En segundo lugar y como una consecuencia concatenada del análisis de la primera sección, se intentará esbozar una definición de la tan citada noción de «discriminación estructural» en virtud de que como se demostrará, la jurisprudencia mayoritaria de la Corte Interamericana de Derechos Humanos no ha sido clara sobre el contenido de dicho concepto. Al respecto y si bien excede el objeto del presente trabajo, se 
debe destacar que el hecho de que se califique una situación de violación a derechos humanos como discriminación estructural trae aparejada una serie de consecuencias jurídicas como, por ejemplo, medidas de reparación más específicas.

Así, la esencia detrás del presente artículo es doble. Por un lado, se intentará esbozar una definición del concepto de «discriminación estructural» que les permita a los lectores utilizarla como una especie de barómetro a los efectos de identificar si una situación constituye una discriminación estructural. No obstante, además interesa de sobremanera que esa definición no se quede como «letra muerta» en el artículo, sino que se aplique a una situación concreta en un ámbito geográfico determinado. Así, teniendo en cuenta tanto la crisis de gobernabilidad migratoria que aqueja al mundo entero como la llamada de atención efectuada por la alta comisionada, se ha seleccionado como caso de estudio a las personas migrantes en Argentina con el objetivo de intentar comprobar o desechar si esta población es víctima de discriminación estructural.

$\mathrm{Al}$ respecto, se deben explicitar algunas consideraciones previo a analizar cada uno de los tres acápites propuestos. En efecto, en Argentina existen algunos antecedentes claramente discriminatorios en contra de las personas migrantes que datan de casi dos décadas atrás (Novick, 200o). Las personas migrantes en Argentina sobre todo las provenientes de Bolivia, Paraguay y Perú- fueron tratadas como una amenaza al trabajo, la sanidad y la seguridad, retórica que terminó plasmándose en prácticas represivas y persecutorias dirigidas a la migración de los países limítrofes, basándose en el Decreto de Ley General 22.439 de Migraciones y de Fomento de la Inmigración - denominada Ley Videla - y mantenida hasta el año 2003 (Domenech, 2007: 76).

Si bien luego de esa legislación se adoptó la Ley 25.871 que vino a cambiar el paradigma en virtud del cual el Estado abordada la temática migratoria (Ceriani, 2004), lo cierto es que ese cambio se mantuvo hasta 2016, cuando se comenzaron a adoptar políticas públicas regresivas en materia de derechos humanos hasta el dictado del Decreto de Necesidad y Urgencia 70/2017, de dudosa constitucionalidad y convencionalidad. ${ }^{2}$

Al respecto, cabe destacar que la Cámara Nacional de Apelaciones en lo Contencioso Administrativo Federal declaró la inconstitucionalidad del mencionado decreto, ${ }^{3}$ principalmente por dos razones: constituir una situación de apropiación de facultades legislativas sin causa constitucional legitimante y porque algunas de sus disposiciones se encuentran en clara contradicción con las normas constitucionales y convencio-

2. Para más información, véase Carril (2017) y el Informe Técnico DNU 70/17 de la Defensoría del Pueblo de la Ciudad Autónoma de Buenos Aires, Argentina, disponible en https://bit.ly/2rBRrDu.

3. Cámara de Apelaciones en lo Contencioso Administrativo Federal, Sala V, Centro de Estudios Legales y Sociales y otros c/ EN-DNM s/ amparo Ley 16.986, 22 de marzo de 2018. 
nales. Sin perjuicio de esto, en el presente artículo no será analizada la decisión de la Cámara de Apelaciones, sino que se centrará en el contenido del DNU 70/2017.

Como se ha adelantado, en este contexto se intentará explorar si se puede hablar de la existencia de una discriminación estructural en contra de las personas migrantes en Argentina que se ha "cristalizado» con el dictado del Decreto de Necesidad y Urgencia 70/2017, el cual establece una relación directa entre la migración y el delito, especialmente el narcotráfico. Por cuestiones de extensión, el análisis del tercer apartado será sobre las políticas públicas argentinas en general y no un análisis minucioso sobre la legislación vigente en materia de migración (Giustiniani, 2004).

\section{Igualdad y no discriminación en el Sistema Interamericano}

Si bien el principio de igualdad y no discriminación pareciera ser simple de conceptualizar, lo cierto es que se han dado una serie de discusiones en torno a su definición (Uprimny Yepes y Sánchez Duque, 2014: 581; Bayefsky, 1990) que exceden el marco del presente artículo. Con el objeto de sortear las diferentes argumentaciones al respecto, en función de lo anterior utilizaré las definiciones del Sistema Interamericano.

La Convención Americana sobre Derechos Humanos posee varios artículos que hacen referencia a la «igualdad»: el artículo 8.2 (garantías judiciales), el artículo 17.4 y 5 (protección de la familia) y el artículo 23.1.b y c (derechos políticos). Sin perjuicio de ello, en su texto existen dos referencias directas y diferentes a la igualdad y no discriminación en los artículos 1.1 y 24.

$\mathrm{Al}$ respecto se puede realizar una primera aproximación sistémica, analizando el lugar que ocupan dentro de la diagramación de la Convención Americana sobre Derechos Humanos. La primera disposición se encuentra dentro de la primera parte de la Convención, donde se enuncian los deberes de los Estados, mientras que el artículo 24 de la Convención Americana sobre Derechos Humanos se encuentra dentro de los derechos civiles y políticos recogidos por la Convención.

En este sentido, a partir del caso Apitz Barbera y otros con Venezuela ${ }^{4}$ la Corte Interamericana comenzó a diferenciar ambas disposiciones, ya que con anterioridad se las había considerado como parte de un mismo esquema dentro del derecho a la igualdad. ${ }^{5}$ En la decisión mencionada, la Corte Interamericana argumentó que la principal diferencia radicaba en que el artículo 1.1 poseía una obligación general que se refería al deber del Estado de respetar y garantizar «sin discriminación» los derechos contenidos en la Convención Americana sobre Derechos Humanos, mientras que la disposición del artículo 24 se aplica para los casos en los que existe una pro-

4. Caso Apitz Barbera y otros, Corte Interamericana de Derechos Humanos, párrafo 209.

5. Véase Ferrer Mac-Gregor y Pelayo Möller (2014: 56) y la Opinión Consultiva OC-18/03 de la Corte Interamericana de Derechos Humanos, párrafo 83 y siguientes. 
tección desigual de la ley interna, ${ }^{6}$ incluyendo todas las leyes que apruebe el Estado y su aplicación. ${ }^{7}$

Si se tiene en cuenta esta diferencia y se utiliza el planteo de Bayefsky relacionado con las cláusulas autónomas y cláusulas subordinadas de igualdad (1990: 3-5), se podría afirmar que el artículo 1.1 constituye una cláusula subordinada en tanto se consagra a la igualdad como una garantía accesoria a los derechos reconocidos en la Convención Americana sobre Derechos Humanos. De hecho, así ha sido utilizada esta disposición por la Corte Interamericana de Derechos Humanos en diversa jurisprudencia, analizando la cláusula de no discriminación del artículo 1.1 en conjunto con otros artículos para determinar la vulneración de los derechos humanos de las mujeres ${ }^{8}$ pueblos indígenas, ${ }^{9}$ personas $\mathrm{LGBTI}^{10}{ }^{10}$ personas con discapacidad ${ }^{11}$, y las que nos interesan a los efectos de este artículo, es decir, las personas migrantes. ${ }^{12}$

Por su parte, el artículo 24 constituiría una cláusula autónoma de igualdad en tanto derecho independiente que se encuentra garantizado en sí mismo y no atado a otro derecho que se encuentre en peligro de ser vulnerado. La Corte Interamericana de Derechos Humanos también lo ha entendido de esta manera y ha declarado su violación en casos relacionados con derechos políticos, ${ }^{13}$ pueblos indígenas, ${ }^{14}$ personas LGBTI ${ }^{15}$ personas migrantes ${ }^{16}$ e investigaciones judiciales de homicidios de mujeres con perspectiva de género. ${ }^{17}$

6. Caso Apitz Barbera y otros con Venezuela, Corte Interamericana de Derechos Humanos, párrafo 209; Caso Flor Freire con Ecuador, Corte Interamericana de Derechos Humanos, párrafo 112; entre otros.

7. Caso Atala Riffo y niñas con Chile, Corte Interamericana de Derechos Humanos.

8. Caso González y otras («Campo Algodonero») con México, Corte Interamericana de Derechos Humanos, párrafo 339-402; Caso Gonzales Lluy y otros con Ecuador, Corte Interamericana de Derechos Humanos, párrafo 291 (en este último caso se hizo mención a su condición de salud, económica y de ser niña como factores de discriminación interseccional).

9. Caso Comunidad Indígena Xákmok Kásek con Paraguay, Corte Interamericana de Derechos Humanos, párrafo 274-275 y punto resolutivo 9.

10. Caso Duque con Colombia, Corte Interamericana de Derechos Humanos, párrafo 138; Caso Flor Freire con Ecuador, párrafo 127 y siguientes.

11. Caso Furlan y familiares con Argentina, Corte Interamericana de Derechos Humanos, párrafo 268-269.

12. Caso Nadege Dorzema y otros con República Dominicana, Corte Interamericana de Derechos Humanos, párrafo 238; Caso Personas dominicanas y haitianas expulsadas con República Dominicana, Corte Interamericana de Derechos Humanos, párrafo 276.

13. Caso Yatama con Nicaragua, Corte Interamericana de Derechos Humanos, párrafo 229.

14. Caso Norín Catrimán y otros (Dirigentes, miembros y activista del Pueblo Indígena Mapuche) con Chile, Corte Interamericana de Derechos Humanos, párrafo 228.

15. Caso Atala Riffo y Niñas con Chile, Corte Interamericana de Derechos Humanos, párrafo 146.

16. Caso de las niñas Yean y Bosico con República Dominicana, Corte Interamericana de Derechos Humanos, párrafo 131-174.

17. Caso Véliz Franco y otros con Guatemala, Corte Interamericana de Derechos Humanos, párrafo 216; 
En este punto resulta fundamental analizar la definición de «discriminación», que si bien no se encuentra esbozada en la Convención Americana sobre Derechos $\mathrm{Hu}$ manos directamente, la Corte Interamericana de Derechos Humanos ha dicho que es:

Toda distinción, exclusión, restricción o preferencia que se base en determinados motivos, como la raza, el color, el sexo, el idioma, la religión, la opinión política o de otra índole, el origen nacional o social, la propiedad, el nacimiento o cualquier otra condición social, y que tengan por objeto o por resultado anular o menoscabar el reconocimiento, goce o ejercicio, en condiciones de igualdad, de los derechos humanos y libertades fundamentales de todas las personas. ${ }^{18}$

Todo esto teniendo en cuenta distintos tratados de derechos humanos.

Si bien la condición migratoria no está específicamente prevista en la definición anterior, la Corte Interamericana de Derechos Humanos la ha empleado cuando ha conocido casos relacionados con personas migrantes, declarando la vulneración de la cláusula subordinada de no discriminación. ${ }^{19}$

$\mathrm{Al}$ respecto, resulta interesante mencionar la Convención Interamericana contra el Racismo, la Discriminación Racial y Formas Conexas de Intolerancia, que contiene en su artículo 1.1 una definición de discriminación e incluye a la condición migratoria entre las categorías prohibidas de discriminación.

En este marco se analizará qué se entiende por discriminación estructural a los efectos de poder corroborar - o no- la existencia de este tipo de discriminación en contra de las personas migrantes en Argentina.

\section{Discriminación estructural}

La Corte Interamericana de Derechos Humanos se ha referido a la discriminación estructural en algunas decisiones, siendo la más reconocida la del caso Campo Algodonero, en el cual el Estado mexicano reconoció la existencia de una situación de discriminación estructural contra las mujeres en México, ${ }^{20} \mathrm{y}$ si bien se hace mención al patrón sistemático de violencia, asesinato y discriminación contra mujeres y niñas

Caso Velásquez Paiz y otros con Guatemala, Corte Interamericana de Derechos Humanos, párrafo 199.

18. «Identidad de género, e igualdad y no discriminación a parejas del mismo sexo. Obligaciones estatales en relación con el cambio de nombre, la identidad de género, y los derechos derivados de un vínculo entre parejas del mismo sexo (interpretación y alcance de los artículos 1.1, 3, 7, 11.2, 13, 17, 18 y 24 , en relación con el artículo 1 de la Convención Americana sobre Derechos Humanos)», Corte Interamericana de Derechos Humanos, Opinión Consultiva OC-24/17, párrafo 62.

19. Caso Nadege Dorzema y otros con República Dominicana, Corte Interamericana de Derechos Humanos, párrafo 238; Caso de Personas dominicanas y haitianas expulsadas con República Dominicana, Corte Interamericana de Derechos Humanos, párrafo 276.

20. Caso González y otras con México, Corte Interamericana de Derechos Humanos, párrafos 129, 152, $450,451$. 
de Ciudad Juárez debido a estereotipos y fenómenos sociales y culturales, ${ }^{21}$ la Corte no definió su significado.

Posteriormente, el tribunal interamericano hizo mención nuevamente a la discriminación estructural, pero esta vez por la orientación sexual de las víctimas, ${ }^{22}$ resaltando que algunos de los actos discriminatorios analizados en el caso se relacionaban con la reproducción de estereotipos asociados a la discriminación estructural e histórica sufrida por las minorías sexuales. En este caso, la Corte nuevamente deja sin definir el término en cuestión, aunque agrega el término «histórico» a su análisis. ${ }^{23}$

Relacionado específicamente con la discriminación en contra de migrantes, la Corte tuvo una primera oportunidad de expedirse sobre una situación de discriminación estructural en República Dominicana, sin embargo, consideró que esto no era necesario, ${ }^{24}$ aunque sí se refirió de manera extensa al contexto de discriminación en contra de migrantes haitianos.

Sin perjuicio de lo anterior, en otro caso contra República Dominicana la Corte hizo mención del contexto histórico en el que se enmarcaban los hechos, ${ }^{25}$ concluyendo que para la época existía una situación en la que las personas haitianas sufrían con frecuencia tratos peyorativos o discriminatorios, inclusive por autoridades estatales. ${ }^{26} \mathrm{~A}$ su vez, la Corte advirtió la existencia de un patrón sistémico de expulsiones que obedecía a una concepción discriminatoria.

En un reciente fallo, la Corte Interamericana ha hecho mención a la discriminación estructural sufrida por trabajadores brasileños que habían sido víctimas de trabajo esclavo, ${ }^{27}$ situación que tenía orígenes históricos y era conocida por Brasil desde al menos $1995 .{ }^{28}$ En este punto resulta interesante traer a colación el voto razonado del juez Ferrer Mac-Gregor Poisot, quien afirmó que los Estados deben considerar la

21. Caso González y otras con México, Corte Interamericana de Derechos Humanos, párrafo 109-462.

22. Caso Atala Riffo y Niñas con Chile, Corte Interamericana de Derechos Humanos, párrafos 92, 93 y 267.

23. Esta idea fue reiterada, casi idénticamente, en dos casos relacionados con derechos de personas LGBTI: Caso Duque con Colombia, Corte Interamericana de Derechos Humanos, párrafo 123; Caso Flor Freire con Ecuador, Corte Interamericana de Derechos Humanos, párrafo 124.

24. Caso Nadege Dorzema y otros con República Dominicana, Corte Interamericana de Derechos Humanos, párrafo 40.

25. Caso de Personas dominicanas y haitianas expulsadas con República Dominicana, Corte Interamericana de Derechos Humanos, párrafos 153-171.

26. Para así decidir, la Corte Interamericana utilizó distintos medios de prueba: informes de organizaciones de la sociedad civil (como amnistía internacional o human rights watch), del banco mundial, observaciones finales de los órganos de tratados, peritos expertos, entre otros.

27. Caso Trabajadores de la Hacienda Brasil Verde con Brasil, Corte Interamericana de Derechos Humanos, párrafo 334 y siguientes.

28. Caso Trabajadores de la Hacienda Brasil Verde con Brasil, Corte Interamericana de Derechos Humanos, párrafo 339 . 
existencia de situaciones sistemáticas de discriminación que en ocasiones responden a contextos específicos e institucionales de negación de derechos humanos ${ }^{29}$ identificando una serie de elementos que permitirían afirmar la existencia de discriminación estructural: un grupo de personas con características inmutables de manera voluntaria o relacionadas a factores históricos de prácticas discriminatorias, aun cuando sea un grupo mayoritario, grupos que se han encontrado en una situación sistémica e histórica de exclusión que les impide acceder a condiciones básicas de desarrollo humano. ${ }^{30}$

En relación con esta temática, Abramovich afirmó que en la jurisprudencia interamericana se produjo un avance hacia un concepto de igualdad material o estructural «que parte del reconocimiento de que ciertos sectores de la población están en desventaja en el ejercicio de sus derechos por obstáculos legales o fácticos y requieren, por consiguiente, la adopción de medidas especiales de equiparación», implicando la necesidad de un trato diferenciado cuando se trata de un grupo desaventajado (2009: 21).

Entonces, ¿qué se debe entender por discriminación estructural? Creo que una aproximación a su definición - tomando en cuenta la jurisprudencia interamericana- consistiría en afirmar que la discriminación estructural es una distinción, exclusión o restricción de los derechos humanos y libertades fundamentales de un grupo histórico y socialmente en desventaja, que comparte características inmutables o inmodificables por la propia voluntad de las personas que lo integran, cuya restricción es causada como resultado de una situación de exclusión social o de «sometimiento» de grupos vulnerables, en forma sistemática y debido a complejas prácticas sociales, prejuicios y estereotipos.

En este sentido, se advierte que el rasgo común que identifica a los grupos históricamente excluidos o en desventaja es que existe una historia de discriminación, de perjuicios negativos que en muchas ocasiones son reforzados por normas internas.

Por esto y a los efectos de poder afirmar o negar que las personas migrantes en Argentina son víctimas de discriminación estructural, a continuación se hará mención de las políticas públicas y de las prácticas relacionadas con esta temática que han tenido lugar en las últimas décadas en Argentina.

\section{Políticas públicas regresivas en Argentina}

En el presente acápite realizaré un sucinto análisis de algunas de las políticas migra-

29. Caso Trabajadores de la Hacienda Brasil Verde con Brasil, Corte Interamericana de Derechos Humanos, voto razonado del juez Ferrer Mac-Gregor Poisot, párrafo 56.

30. Caso Trabajadores de la Hacienda Brasil Verde con Brasil, Corte Interamericana de Derechos Humanos, voto razonado del juez Ferrer Mac-Gregor Poisot, párrafo 80. 
torias decretadas por el gobierno argentino con el objetivo de intentar demostrar la práctica del Estado al respecto.

$\mathrm{El} 3$ de mayo de 2016 se anunció el endurecimiento de las políticas migratorias que se planeaba llevar a cabo con el objetivo de «luchar contra el tráfico de personas, el narcotráfico, el terrorismo y el trabajo en negro o esclavo al que se somete a los inmigrantes irregulares». ${ }^{31}$ Entre las medidas previstas, el director nacional de Migraciones afirmó que se incrementarían los planteles y la capacitación de inspectores en los controles de permanencia de extranjeros para impedir la irregularidad migratoria.

$\mathrm{Al}$ respecto, no se debe perder de vista que toda política migratoria que adopte un Estado debe estar encaminada a la protección y regularización de las personas migrantes en situación irregular, ${ }^{32}$ y no a su castigo. Sin perjuicio de esto, luego del anuncio del director nacional se llevaron a cabo una serie de medidas regresivas en materia de derechos humanos de las personas migrantes. ${ }^{33}$

Entre esas medidas se debe hacer mención al Decreto 956/2016, artículo 1, que introdujo un aumento de las tarifas migratorias incrementando las tasas para residencias permanentes en más de un 150\%, aun cuando el Comité sobre los Derechos de los Trabajadores Migratorios ya había recomendado a Argentina en 2011 que redujera las tasas administrativas. ${ }^{34}$

En lo que hace a las expulsiones de las personas migrantes, según el informe presentado por Organizaciones de la Sociedad Civil ante el Comité para la Eliminación de la Discriminación Racial, en 2014 fueron dictadas 1760 expulsiones, en 2015 fueron 1908 y hasta septiembre de 2016 este número ya estaba en 3258 , representando un aumento de $70 \%$ en relación con el año anterior. ${ }^{35}$

Estas medidas, tomadas durante 2016, sirvieron de base para la modificación de la legislación migratoria que llegó un año más tarde.

El 27 de enero de 2017 se dictó el Decreto de Necesidad y Urgencia 70/2017, el cual introduce una serie de cambios significativos a la Ley 25.871 que se encuentran en clara contradicción con los estándares internacionales de derechos humanos, sobre todo con el Objetivo 17 del Pacto Mundial que estipula la necesidad de eliminar toda

31. Martín Dinatale, «El Gobierno prevé endurecer los controles y la política migratoria», La Nación, 3 de mayo de 2016, disponible en https://bit.ly/2FotMzu.

32. Artículos 69 y 79 de la Convención Internacional sobre la Protección de los Derechos de todos los Trabajadores Migratorios y de sus Familiares.

33. Para un análisis exhaustivo de las políticas públicas regresivas en materia de derechos humanos de las personas migrantes, véase Olivera (2017).

34. Observaciones finales del Comité de Protección de los Derechos de Todos los Trabajadores Migratorios y de sus Familiares, Argentina, párrafo 34.

35. «Informe sobre Argentina, Situación de los derechos humanos de las personas migrantes», Abogados y abogadas del Noroeste Argentino en Derechos Humanos y Estudios Sociales (ANDHES) y otros, disponible en https://bit.ly/2Qrn8ro. 
forma de discriminación, comprometiéndose los Estados a promover un discurso basado en evidencias empíricas que generen una percepción más realista, humana y constructiva con respecto a la migración.

Sin perjuicio de esta disposición, de la lectura de los considerandos del DNU se evidencia que se intenta establecer una relación entre la migración y la lucha contra el narcotráfico, ${ }^{36}$ creando la idea - errónea- de que el endurecimiento de las políticas migratorias traería aparejado una disminución del delito. Al respecto, el 24 de enero de 2017 la ministra de Seguridad Patricia Bullrich afirmó que era necesario ordenar las relaciones con Paraguay, Bolivia y Perú - mismas nacionalidades que han sido históricamente criminalizadas- porque el 33\% de los presos por narcotráfico eran extranjeros..$^{37}$ Sin embargo, esa cifra se refiere solamente a las cárceles del Sistema Penitenciario Federal y no al total de la población carcelaria, manipulándose los datos existentes a los efectos de crear una justificación para la criminalización de las personas migrantes..$^{38}$

Como se ha adelantado, estas políticas públicas no son novedosas, ya que las personas migrantes han sido históricamente discriminadas en Argentina. También fueron estigmatizadas como la «invasión silenciosa» en la revista La Primera de la Semana en una publicación del 4 de abril de 2000, donde se afirmó lo siguiente: «los extranjeros ilegales son más de 2 millones. Les quitan trabajo a los argentinos. Usan hospitales y escuelas. No pagan impuestos. Algunos delinquen para no ser deportados. Los políticos miran para otro lado».

A lo anterior se le debe sumar que el Sistema Universal de Protección de Derechos Humanos ha resaltado esta problemática que se suscita en Argentina en relación con los derechos humanos de las personas migrantes relacionados con su derecho de de-

36. También observado por el Comité de Protección de los Derechos de Todos los Trabajadores Migratorios y de sus Familiares: Observaciones finales del Comité de Protección de los Derechos de Todos los Trabajadores Migratorios y de sus Familiares, 2 de noviembre de 2011, CMW/C/ARG/CO/1, párrafo 17 y 18.

37. «Controles migratorios: Patricia Bullrich vinculó a los extranjeros con el narcotráfico y pidió «ordenar las relaciones con Paraguay, Perú y Bolivia»", Infobae, 24 de enero de 2017, disponible en https:// bit.ly/2EUNtcb.

38. Para un mayor análisis, véase Cámara de Apelaciones en lo Contencioso Administrativo Federal, Sala V, «Centro de Estudios Legales y Sociales y otros c/ EN-DNM s/ amparo Ley 16.986», 22 de marzo de 2018, voto del doctor Treacy, considerando V.4. 
fensa en juicio, ${ }^{39}$ acceso a políticas sociales, ${ }^{40}$ discriminación xenófoba, ${ }^{41}$ entre otras problemáticas.

Por último, el Comité para la Eliminación de la Discriminación Racial en las observaciones finales a Argentina reconoció la existencia de una discriminación estructural de las personas migrantes, particularmente aquellos en situación irregular, resaltando la falta de acceso a servicios básicos..$^{42}$ En un apartado específico sobre la situación de los migrantes, el Comité - haciendo uso de la recomendación general 30 sobre la discriminación contra los no ciudadanos- llamó la atención a Argentina para que vele porque no se introduzcan prácticas y normas que representen un retroceso en relación con la normativa vigente. ${ }^{43}$

\section{Conclusión}

Este artículo ha intentado contribuir en un doble sentido. Por un lado, en identificar las características del concepto «discriminación estructural» para intentar aproximarse a una definición. Por otra parte, se ha intentado demostrar que se puede realizar una especie de test, teniendo en mente esa definición, a los efectos de identificar si un grupo particular en un contexto geográfico determinado es víctima de una situación de discriminación estructural.

En relación puntual al fenómeno migratorio, diversos autores han reconocido que en Argentina ha habido distintas fórmulas de exclusión a lo largo de toda la historia migratoria, siendo el Estado uno de los principales promotores (Monclús Masó y García, 2012: 323-365; Domenech, 2007; Novick, 2000).

Desde hace más de dos décadas que las personas migrantes en Argentina -sobre todo las provenientes de Bolivia, Paraguay y Perú - han sido tratadas como una amenaza al trabajo, la sanidad y la seguridad, lo que se ha visto reflejado no solo en la legislación interna, sino también en la prensa de la época. En este sentido, el Sistema Universal de Protección de Derechos ha llamado la atención sobre esta situación,

39. «Informe del Grupo de Trabajo sobre la Detención Arbitraria acerca de su misión a la Argentina», Grupo de Trabajo sobre la Detención arbitraria, 19 de julio de 2018, A/HRC/39/45/Add.1, párrafo 6567; «Observaciones finales sobre los informes periódicos quinto y sexto combinados de la Argentina», Comité contra la Tortura, 24 de mayo de 2017, CAT/C/ARG/CO/5-6, párrafo 33-34.

40. "Observaciones finales sobre el cuarto informe periódico de Argentina», Comité de Derechos Económicos, Sociales y Culturales, 12 de octubre de 2018, E/C.12/ARG/CO/4, párrafo 26-27.

41. «Observaciones finales del Comité para la Eliminación de la Discriminación Racial», Comité para la Eliminación de la Discriminación Racial, 27 de abril de 2001, CERD/C/304/Add.112, párrafo 13.

42. "Observaciones finales sobre los informes periódicos 21 a 23 combinados de la Argentina», Comité para la Eliminación de la Discriminación Racial, párrafo 6.

43. «Observaciones finales sobre los informes periódicos 21 a 23 combinados de la Argentina», Comité para la Eliminación de la Discriminación Racial, párrafo 33-34. Por otra parte, se debe tener en cuenta que, al momento de la revisión, Argentina no había adoptado el DNU 70/2017. 
resaltando la discriminación de la que son víctimas las personas migrantes, llegando a catalogarla como discriminación estructural.

A su vez, se advierte que en los últimos años se han adoptado políticas que progresivamente han llevado a las personas migrantes a «desaparecer» de Argentina, generando un aumento de las tasas migratorias que imposibilite su regularización y, en el caso de que logren acceder a ella, aumentando la cantidad de expulsiones decretadas. Ahora bien, si nada de esto es suficiente, el DNU vino a terminar de socavar los derechos humanos de las personas migrantes, cuanto menos de los provenientes de Paraguay, Bolivia y Perú, países que según el Ministerio de Seguridad son los Estados con los cuales se deben «ordenar las relaciones» debido a que - según sus dichos, sin pruebas fácticas- el $33 \%$ de las personas privadas de libertad eran extranjeros.

A esto se le debe adicionar el prejuicio que se encuentra positivizado en el DNU $70 / 2017$, cuyos considerandos establecen una relación entre la migración y la lucha contra el narcotráfico, nuevamente sin pruebas fácticas que lo sustenten, creando la idea - errónea - de que el endurecimiento de las políticas migratorias traería aparejado una disminución del delito.

En este contexto, se puede deducir lógicamente que las personas migrantes en Argentina provenientes de Paraguay, Bolivia y Perú, constituyen un grupo histórico y socialmente en desventaja que comparten como característica inmutable la condición migratoria, categoría reconocida expresamente como sospechosa por la Convención Interamericana contra el Racismo, la Discriminación Racial y Formas Conexas de Intolerancia.

Por último, se puede afirmar que la distinción de este grupo ha tenido por objeto y resultado el menoscabo del goce de derechos humanos como la libertad de circulación, la protección a la familia-especialmente la reunificación familiar-, las garantías judiciales y el derecho al debido proceso, entre otros derechos, que se han visto gravemente menoscabados con la adopción del DNU, positivización de la discriminación estructural en Argentina en contra de las personas migrantes.

\section{Referencias}

Aвramovich, Víctor E. (2009). «De las violaciones masivas a los patrones estructurales: Nuevos enfoques y clásicas tensiones en el sistema interamericano de derechos humanos». Revista SUR, 6 (11): 7-39. DOI: 10.1590/S1806-64452009000200002.

BAYEFSKY, Anne (1990). «The Principle of Equality or Non-Discrimination in International Law». Human Rights Law Journal, 11 (1-2): 1-34. Disponible en https://bit. $1 y / 365 \mathrm{e} 6 \mathrm{Hq}$.

Ceriani, Pablo (2004). «Nueva Ley: Un paso hacia una concepción distinta de la migración». En Rubén Giustiniani (compilador), Migración: Un derecho humano 
(pp. 113-135). Buenos Aires: Prometeo.

Domenech, Eduardo E. (2007). «La agenda política sobre migraciones en América del sur: el caso de la Argentina». Revue Européenne des Migrations Internationals, 23 (1): 71-94. DOI: 10.4000/remi.3611.

Ferrer Mac-Gregor, Eduardo y Carlos María Pelayo Möller (2014). "Artículo 1. Obligación de respetar los derechos». En Steiner Cristian y Patricia Uribe (coordinadores), Convención Americana sobre Derechos Humanos comentada (pp. 579605) Bogotá: Konrad-Adenauer-Stiftung.

Giustiniani, Rubén. (2004). Migración, un derecho humano: Ley de migraciones 25.871. Buenos Aires: Prometeo.

Monclús Masó, Marta y María Bernarda García (2012). «El impacto de las migraciones en la criminalidad en la Argentina: mitos y realidades». En El impacto de las migraciones en Argentina (pp. 323-365), Buenos Aires: OIM.

Novick, Susana (200o). «Políticas migratorias en la Argentina». En Enrique Oteiza, Susana Novick y Roberto Aruj (editores), Inmigración y discriminación. Políticas y discursos (pp. 87-166). Buenos Aires: Grupo Editor Universitario.

Olivera, Martina (2017). «Migrantes en la República Argentina del 2016-2017: una perspectiva desde el Derecho Internacional de los Derechos Humanos». Actas de las III Jornadas de Jóvenes Investigadores en Ciencias Sociales.

Uprimny Yepes, Rodrigo y Luz María Sánchez Duque (2014). «Artículo 24. Igualdad ante la Ley». En Steiner Cristian y Patricia Uribe (coordinadores), Convención Americana sobre Derechos Humanos comentada (pp. 579-605) Bogotá: Konrad-Adenauer-Stiftung.

Vera-Espinoza, Marcia, Leiza Brumat y Andrew Geddes (2017). «Migration Governance in South America: Where is the region heading?». MPC Blog. Disponible en https://bit.ly/2Qp6TLx.

\section{Sobre la autora}

Martina Olivera es abogada por la Universidad de Buenos Aires e Investigadora de proyectos UBACyT y DECyT. Ha sido investigadora ad-honorem del Instituto y Justicia y Derechos Humanos de la Universidad Nacional de Lanús, así como del Consorcio Latinoamericano de Posgrado en Derechos Humanos en temáticas relacionadas con derecho internacional de los derechos humanos. Ha sido becaria de la Fundación Fulbright para cursar estudios cortos en la Universidad de Massachussets y de la Fundación Konrad Adenauer Stiftung para realizar una visita de Investigación en Heidelberg, Alemania. Ha sido asesora sobre Sistema Interamericano y Universal de Protección de Derechos Humanos en la Secretaría de Derechos Humanos de la Provincia de Buenos Aires, consultora de la Oficina Regional del Alto Comisionado de Naciones Unidas para los Derechos Humanos (ACNUDH), así como de la Aso- 
ciación Internacional de Lesbianas, Gays, Bisexuales, Trans e Intersex-ILGA. Por otra parte, se ha desempeñado como visitante profesional en la Corte Interamericana de Derechos Humanos. Su correo electrónico es martinaolivera@gmail.com. 